\title{
Application of conducting polymer layer for measurement of Ag nanoparticle concentration using surface plasmon resonance
}

\begin{abstract}
An application of polypyrrole and polypyrrole-chitosan were presented to measure the concentration of silver ion and silver nanoparticles using surface plasmon resonance sensor. These results were compared to those for atomic absorption spectroscopy method. The sensing layers were prepared using an electrodeposition on gold layer with the sensor limitation at about $1 \mathrm{mg} / \mathrm{L}$, and well correlated to prior atomic absorption spectroscopy results. The sensor with a polypyrrole-chitosan layer was more sensitive and accurate than the sensor with just a polypyrrole layer.
\end{abstract}

Keyword: Laser ablation; Monoolein; Polypyrrole; Polypyrrole chitosan; SPR sensor 